\title{
Empowering telemedicine - Essential for neurologic and ophthalmologic conditions in the context of a pandemic and beyond
}

\author{
Vlad-Ioan Suciu', Corina-Iuliana Suciu'2,3, Simona Delia Nicoara ${ }^{2,4}$, \\ Lacramioara Perju-Dumbrava ${ }^{1}$ \\ ${ }^{1}$ Department of Neuroscience, "Iuliu Hatieganu" University of Medicine and Pharmacy, \\ Cluj-Napoca, Romania \\ ${ }^{2}$ Department of Ophthalmology, Emergency County Hospital, Cluj-Napoca, Romania \\ ${ }^{3}$ University of Oradea, Romania \\ ${ }^{4}$ Department of Ophthalmology, "Iuliu Hatieganu" University of Medicine and Pharmacy, \\ Cluj-Napoca, Romania
}

\begin{abstract}
In this paper we discuss the importance of telemedicine in two parts - first, regarding two of the common and disabling nervous system disorders: Parkinson's disease and stroke; and the second part, regarding the screening for diabetic retinopathy. We also debate on possible links between these medical conditions. More and more connections between pathologies are discovered every day; sometimes unimagined. In order to prevail over the diseases causing today's burden, we must work together, collaborate and understand that there could be no boundaries in the complexity of diseases.

With the recent progress in medicine, but also in telecommunication, the remote diagnosis, screening and monitoring of illnesses, is now up-to-date more than ever. It is our task to embrace the future perspectives and to adapt in order to benefit from these concepts. We believe that through telemedicine, more patients will have access to quality healthcare services and ultimately improve their quality of life.
\end{abstract}

Keywords: telemedicine, computer interface, network, machine learning, Parkinson's disease, stroke, diabetic retinopathy, tele-neurology, tele-ophthalmology

\section{INTRODUCTION}

Since the widespread of computer networking in the 1980's, more and more people have had access to the internet. The concept of remote work has developed in a fast pace thanks to the modern, highspeed network connections. Telemedicine is a relatively novel concept involving the remote, real-time diagnosis and management of patients by using computer interfaces through secured internet connections. Telemedicine has been developed for areas where there are important restrictions regarding the fast access of emergency healthcare providers, such as rural areas (1-6).
The newest healthcare crisis, secondary to the coronavirus pandemic in 2020, opens a new perspective on the great potential of the broad applications of telemedical services, not only in restricted access areas, but also in most hospitals. Telemedicine with all its branches could have beneficial effects during the pandemic and beyond. Tele-neurology, tele-ophthalmology, tele-radiology are some of the medical specialties which can adapt to telemedical needs (7-10).

Tele-neurology (TN), an important branch of telemedicine, is now in full development and has a great potential of adaptation to the remote health- 
care concept. The neurologic disorders are among the leading causes of disability worldwide and fast access for patients suffering from acute neurological disorders is sometimes impossible. TN has already demonstrated its potential in the acute care of stroke patients (Telestroke), but has not been yet implemented widely in the current mainstream clinical practice. Promoting the broad application of TN in every hospital can increase access of all patients to the highest quality healthcare. The TN concept is not only suitable for emergency situations, but can be applied also for chronic disorders, such as Parkinson's disease, seizure disorders and possibly other pathologies. This is achieved with the help of videoconference and telemonitoring, by applying remote monitor-sensors to patients. Of course, patient consent and data protection policies are imperative in these situations. In this way, medical institutions can become decongested and healthcare could migrate to the patient's home. Patient's quality of life can increase and the anxiety regarding long-hour waiting and consultation in the doctor's office can be eliminated (7).

\section{TELE-NEUROLOGY APPLIED IN PARKINSON'S DISEASE MONITORING}

A national randomized controlled trial (RCT) published in Neurology in 2017, concluded that the remote assistance for Parkinson's disease (PD) patients is possible. Beck and colleagues reported that most doctors $(86 \%)$ and patients $(97 \%)$ evaluated positively the virtual consultations and the patients seemed to prefer the virtual consultations to face-toface visits (55\% vs. $18 \%$; statistical significant results). Despite this, the virtual consultations seemed not to improve the quality of life for the patients. There were no safety issues during the study. The study group discussed the possible advantages of the remote visits in PD patients as the following:

- they eliminate the time consuming face-toface visits by an average of $169 \mathrm{~min}$./visit

- they eliminate the cost of transportation to and from the hospital or office

- they eliminate the need for large hospital spaces and waiting rooms

- they are convenient for patients, who are being evaluated from their homes
- they reduce the number of hospitalizations, thereby reducing the costs

- they seem to be most beneficial for PD patients with advanced disease, in rural areas or possibly those in nursing homes.

The study group authors also recognize the possible limitations concerning the virtual consultations. For some patients, there can be difficulties in using the computer interface or there may be a poor internet connection. Some aspects of the clinical examination could be difficult. There may also be a limitation of care due to the need for multidisciplinarity in complex cases (11).

In 2019, Botros and colleagues emphasized on the need for 24 hour monitoring of PD patients due to its fluctuating nature and highly individual specific nature. Small sensors attached at the wrists, ankles and hip can be worn at home and they can evaluate precisely each patient. This may increase the management efficiency due to medication adaptation under the precise knowledge of the timing of the motor fluctuations (12).

In 2019, Albani and colleagues also approached the subject of remote sensor monitoring of PD. They highlighted the importance of remote sensor monitoring in the future for PD patients because of the projected increase in prevalence of these patients. Their sensor system could analyze 6 different tasks within UPDRS and the study group believes it could be feasible and cheap to implement in the future because of its accuracy (13).

Kleinholdermann and colleagues also stress the importance of remote monitoring of PD evolution through modern mobile technology in order to better control the unpredictable motor fluctuations of the disease. This would increase the precision of the treatment and symptom control (14).

In 2019, Espay and colleagues working at The Movement Disorders Society Task Force on Technology, highlight the need for a consensus in remote monitoring of PD. They worked out a guideline for all healthcare professionals striving to cross the barrier of remote monitoring, in order to improve the symptomatic treatment and improve the quality of life for PD patients. They believe that this recommendation would harmonize the work of professionals in this developing field (15).

Cabrera-Martos and his team proposed the remote evaluation of the motor symptoms of the 
upper limbs in PD patients, as they are more common and they impede functionality the most. They used an application and compared the virtual visit over the internet, to the face-to-face consultation, and ultimately concluded that the remote evaluation had a good reliability of measurement $(\mathrm{p}>$ 0.75) (16).

Prince and de Vos studied the possibility of PD disease severity prediction on 1815 participants by using remote sensors. They tested deep learning algorithms on a finger-tapping test and found that the convolutional network had similar results as the traditional method of disease classification. Their results stress the possibility of remote evaluation of PD by using wearable sensors in conjunction with machine learning method analysis (17).

Rovini and colleagues gathered information from 3 databases from 2008 until 2017 to review the medical literature regarding the telemedicine topic in PD. Because the clinical expression of PD differs from person to person, the need for a personalized therapy is high. Here come into work remote assessment tools, which could have a great objective value in the future (18).

Another recent study on 16 PD patients compared machine learning analysis with neurologist's staging of disease. This revealed that the finger-tapping test and hand opening-closing test delivered statistical significant results (19).

\section{TELE-NEUROLOGY IS SUITABLE FOR BOTH PD AND STROKE}

PD and stroke are common pathologies in the older population. PD patients are not exempt from stroke, and unfortunately PD and stroke can co-exist in the same patient. Levine and colleagues conducted a case-control study on 119 PD patients and 238 patients who were not suffering from PD and concluded that PD does not have a protective effect against ischemic stroke as Struck suggested in an earlier study (20).

Karlijn F. de Laat and colleagues studied 430 patients without PD or dementia and performed cerebral MRI scans in order to quantify the white matter lesions, including lacunar infarcts. They correlated the magnitude of these lesions with the UPDRS-III scale and found that white matter lesions, especially in the parietal and frontal lobes, raised the risk of having mild signs of parkinsonism (21).

Judd and colleagues studied the potential neuroprotective role of vitamin $\mathrm{D}$ in stroke, as its deficiency has been possibly related to stroke, Parkinson's disease and other disorders. Further studies are needed (22).

The implementation of remote healthcare services for monitoring PD progression at home and for the acute treatment of stroke in restricted areas is necessary in establishing a more complete and efficient tele-neurological system.

Solenski published in 2018 a paper regarding the telestroke concept, recognizing that the first such programs were first reported in the 90's. Since then, sustained efforts have been made, in order to establish recommendations for good clinical practice. It has been shown that the face-to-face NIHS scale evaluation had similar results to that of videoconferencing method. Also, rapid decisions regarding acute therapy can be made by the association of remote head CT scan interpretation (23).

In a recent paper, Halbert and Bautista explained the importance of telestroke, which can increase stroke care in access restricted areas, can avoid disability and ultimately improve the quality of life for patients, and is also considered to be cost efficient by decreasing the period of hospitalization and healthcare costs. The authors also recognize some potential disadvantages of telestroke programs, such as difficulties in accessing technology or even financial problems (24).

Campbell also agrees on the fact that telemedical methods are essential in providing better guidance, especially in time dependent and emergency situations such as acute stroke treatments. This can maximize the beneficial effect of the acute treatment (25).

Kane-Gill and Rincon recognize the expansion of telemedical services, which, they believe, can establish uniformity between the disadvantaged, rural hospitals and the important health-care centers of excellence. However, they warn about the insufficient research regarding this aspect of medical care (26).

The telemedicine group fo the AAN published a paper in 2020 recognizing the ever so expanding capability of telemedicine in many neurologic disorders. Telestroke programs have important evi- 


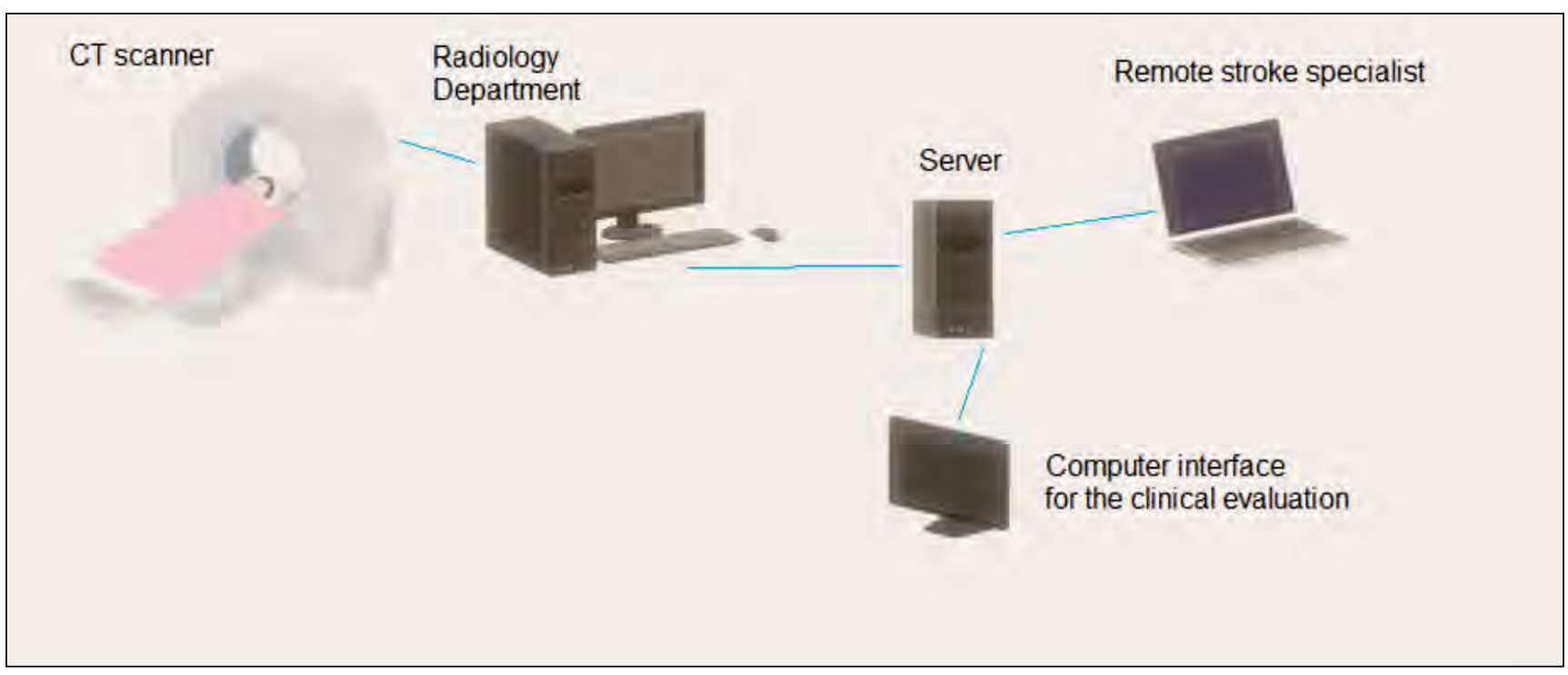

FIGURE 1. Telestroke networking concept, imagined (image edited by the authors with Microsoft Paint $3 D$ on Windows 10)

dence sustaining its value, but also many subspecialities of neurology seem to be non-inferior regarding to the classic face-to-face evaluations done in the office. TN seems to have benefits for the future by reducing healthcare cost, increasing access to specialized evaluation and improving clinical outcomes (27).

\section{TELE-OPHTHALMOLOGY: THE DIABETIC RETINOPATHY AND LINKS TO PD AND STROKE}

A link between PD and diabetes mellitus (DM), including the diabetic retinopathy (DR), has been suggested long ago. Both PD and DM, being common diseases, determine an important impact on the healthcare system through the disabling nature of them and high treatment costs. Pagano and colleagues presented in a recent paper the results of their study in which they compared 25 patients with PD and diabetes mellitus, 25 patients with PD without DM, 14 patients without PD, but with DM and 14 healthy individuals. They evaluated the patients both clinically and with auxiliary methods (striatal dopamine binding and tau-protein CSF levels) and concluded that the association between PD and DM leads to a higher severity of symptoms (28).

Vidal-Martinez and colleagues support the theory that alpha-synuclein has an inhibitory effect on both the insulin secretion in the pancreatic cells and also on the dopamine synthesis in the brain, reveal- ing to us that this could be another link between PD and DM (29).

Diabetes mellitus is one of the most common metabolic disorders worldwide, giving rise to multiple complications, including the diabetic retinopathy (DR), a frequent cause of blindness. We know today that DM is an important vascular risk factor, being often involved in the pathogenesis of ischemic stroke. It has been estimated that the prevalence of diabetes by year 2030 can increase up to $4.4 \%$ (366 million affected persons), making the burden even higher for the healthcare systems. These facts give us a short period of time in which we can act on preventing the increase in incidence of diabetes and consequently, of its complications (DR, renal failure, limb amputations, stroke, myocardial infarctions and many others) (30).

Gioia and Salducci explained in a recent paper the significance of telemedicine in ophthalmology, especially in monitoring the diabetic retinopathy, the retinopathy of prematurity or even macular diseases. The diabetic retinopathy is also compatible with telemedicine in screening programs to help clinicians diagnose and treat, if necessary, the condition in its earliest stages. Telemedicine in ophthalmology looks promising, reduces healthcare costs, can serve as a support platform for clinician collaboration at different sites and is especially justified in the diagnosis and monitoring of eye disorders which require imaging (31). 
Liu and colleagues also discuss in a recent paper the fact that tele-ophthalmology could decrease blindness by better screening individuals with diabetic retinopathy. It is important for healthcare systems to widely ensure the technology for the screening of diabetic retinopathy (32).

Salongcay and Silva published in 2018 a review in which they value the role of telemedicine in diagnosing and managing the diabetic retinopathy (DR). The remote diagnosis of DR was similar in efficiency with the face-to-face clinical exam (sensitivity $62.5 \%$ vs. $98.2 \%$ and specificity $76.6 \%$ vs. $98.7 \%$ ). Because of the major increase in prevalence of diabetic patients until 2030, it will take about 4.5 million hours/year of consultations per ophthalmologist, in order to assess once, every patient. This prediction and the fact that the diabetic complications (DR, including blindness) are preventable if detected early, shows us the great importance to further develop and widely implement remote screening via Tele-ophthalmology for individuals with a risk for diabetic retinopathy (33).

Liesenfeld et al. showed that the diagnosis efficiency of moderate non-proliferative DR and/or macular edema related to DR had a median sensitivity of $85 \%$ and a median specificity of $90 \%$ (CI $95 \%)$. Although these results are encouraging, they warn about the need for combined evaluation of retinal photography with visual acuity measurement in order to improve the quality of diagnosing (screening) DR with macular edema (34).

Another important factor explaining the close collaboration between ophthalmologists and neurologists is the fact that many patients suffering from PD also develop visual disturbances. These can be related to the intrinsic pathology of PD and can include reduced contrast sensitivity, impaired color vision perception, altered visual acuity and others. The pathologic process is still in debate (35).

\section{DISCUSSIONS}

Indeed, since the great progression of technology and network connectivity, the traditional faceto-face medical consultations seem to be outdated. We will probably witness the transfer from hospital healthcare to home healthcare. This is justified because of the vast benefits Telemedicine brings to the patients and also to the healthcare systems. More and more patients can have access to specialized medical consultations. More people can get screened for common disorders and can receive ultimately treatments in the earlier stages of diseases, thereby preventing complications and disability. Also, patients will have a higher comfort if they are consulted in their homes. For the healthcare systems, on the other hand, the cost of treatments will decrease, hospitals will get decongested, and the need for long hospitalizations will decrease.

Neurology and ophthalmology have one of the best potentials in adaptation to Telemedicine, not only for acute disorders, like stroke, but also for chronic disorders, like Parkinson's disease, epilepsy and others. Screening coverage in DR is with the traditional methods insufficient, but with the expansion, through Telemedicine, of the screening of diabetic patients for diabetic retinopathy, will, with no doubt, decrease the incidence and prevalence of blindness.

Another important aspect to point out is the contributions of machine learning in medicine, which can help clinicians take better decisions. Machine learning should probably be used as an important aid in difficult decisions.

Some concerns and limitations to telemedicine may exist, such as too little human contact, data protection issues and consent, but these could be overcome in the future. The benefits clearly overweigh the downsides of telemedicine, through high patient access, 24/7 availability, cost efficiency, no exposure risk (with regard to infections) and applicability in both low- and high-income countries. Telemedicine should also be adapted for collaboration between specialists, since the pathologies are interconnected and our understanding of them increases in complexity every day.

\section{CONCLUSIONS}

By promoting telemedicine in this developing era of medicine with the aim of making healthcare better and more accessible to all people around the world is gratifying. With a better collaboration among physicians of different specialties and IT specialists, telemedicine could be improved and be the future in medicine. 


\section{REFERENCES}

1. Hafner K, Lyon M. Where Wizards Stay Up Late: The Origins of the Internet. New York: Simon \& Schuster; 1998.

2. West J, Andrews J, Dean T. Network+ Guide to Networks. 8th Edition. Boston: Cengage Learning; 2018.

3. Hayiroglu MI. Telemedicine: Current Concepts and Future Perceptions. Anatol J Cardiol. 2019;22:21-2.

4. Cowie MR, Bax J, Bruining N, Cleland JGF, Koehler F, Malik M, et al. e-Health: a position statement of the European Society of Cardiology. European Heart Journal 2016:37(1);63-6.

5. Mashima PA, Doarn CR. Telemedicine and e-Health. Telemedicine Journal and E-Health 2008;14(10):1101-17.

6. Maheu M, Whitten P, Allen A. E-Health, Telehealth, and Telemedicine: A Guide to Startup and Success. New York: John Wiley \& Sons; 2001.

7. Dorsey E, Glidden A, Holloway M, et al. Teleneurology and mobile technologies: The future of neurological care. Nat Rev Neurol. 2018;14:285-297.

8. Boucher MC, Quyn TN, Karine A. Mass community screening for diabetic retinopathy using a nonmydriatic camera with telemedicine. Canadian Journal of Ophthalmology 2005;40(6):734-742.

9. Pérez MA, Bruce BB, Newman NJ, Biousse V. The Use of Retinal Photography in Nonophthalmic Settings and Its Potential for Neurology. The Neurologist 2012; 18(6):350-5.

10. Schreyer AG, Elgharbawy M, Dendl LM, et al. Charakterisierung teleradiologisch untersuchter Patienten an einem Krankenhaus der Schwerpunktversorgung. Radiologe 2020.

11. Beck CA, Beran DB, Biglan KM, Boyd CM, Dorsey ER, Schmidt PN, et al. National randomized controlled trial of virtual house calls for Parkinson disease. Neurology 2017; 89(11):1152-1161.

12. Botros $A$, Schütz $N$, Camenzind $M$, Urwyler $P$, Bolliger $D$, Vanbellingen T, et al. Long-Term Home-Monitoring Sensor Technology in Patients with Parkinson's Disease-Acceptance and Adherence. Sensors 2019;19:5169.

13. Albani G, Ferraris C, Nerino R, Chimienti A, Pettiti G, Parisi F, et al. An Integrated Multi-Sensor Approach for the Remote Monitoring of Parkinson's Disease. Sensors 2019;19:4764.

14. Kleinholdermann U, Melsbach J, Pedrosa DJ. Remote-Messung bei idiopathischem Parkinson-Syndrom. Nervenarzt 2019;90:1232-1238.

15. Espay AJ, Hausdorff JM, Sánchez-Ferro Á, Klucken J, Merola A, Bonato $\mathrm{P}$, et al. A roadmap for implementation of patient-centered digital outcome measures in Parkinson's disease obtained using mobile health technologies. Mov Disord. 2019;34:657-663.

16. Cabrera-Martos I, Ortiz-Rubio A, Torres-Sánchez I, López-López L, Rodríguez-Torres J, Carmen Valenza M. Agreement Between Face-to-Face and Tele-assessment of Upper Limb Functioning in Patients with Parkinson Disease. Journal of Injury, Function and Rehabilitation 2019;11:590-596.

17. Prince J, de Vos M. A Deep Learning Framework for the Remote Detection of Parkinson's Disease Using Smart-Phone Sensor Data. 40th Annual International Conference of the IEEE Engineering in Medicine and Biology Society (EMBC) 2018:3144-3147.

18. Rovini E, Maremmani C, Cavallo F. Automated Systems Based on Wearable Sensors for the Management of Parkinson's Disease at
Home: A Systematic Review. Telemed J E Health. 2019;25(3):167183.

19. Butt AH, Rovini E, Dolciotti C, et al. Objective and automatic classification of Parkinson disease with Leap Motion controller. Biomedical Engineering Online 2018;17(1):168.

20. Levine RL, Jones JC, Bee N. Stroke and Parkinson's disease. Stroke. 1992;23:839-842.

21. de Laat KF, van Norden AGW, Gons RAR, van Uden IWM, Zwiers MP, Bloem BR, et al. Cerebral White Matter Lesions and Lacunar Infarcts Contribute to the Presence of Mild Parkinsonian Signs. Stroke. 2012;43:2574-2579.

22. Judd SE, Le A, Kleindorfer DO, Kissela B, Muntner P, Gutierrez $O$. Vitamin D Intake: A Novel Neuroprotectant? Stroke. 2012;43:A2212.

23. Solenski NJ. Telestroke. Neuroimaging Clin N Am. 2018;28(4):551563.

24. Halbert K, Bautista C. Telehealth Use to Promote Quality Outcomes and Reduce Costs in Stroke Care. Crit Care Nurs Clin North Am. 2019;31(2):133-139.

25. Campbell BC. Advances in stroke medicine. Med J Aust. 2019;210(8):367-374.

26. Kane-Gill SL, Rincon F. Expansion of Telemedicine Services: Telepharmacy, Telestroke, Teledialysis, Tele-Emergency Medicine. Crit Care Clin. 2019;35(3):519-533.

27. Hatcher-Martin JM, Adams JL, Anderson ER, Bove R, Burrus TM, Chehrenama M, et al. Telemedicine in neurology: Telemedicine Work Group of the American Academy of Neurology update. Neurology. 2020;94(1):30-38.

28. Pagano $\mathrm{G}$, Polychronis $\mathrm{S}$, Wilson $\mathrm{H}$, et al. Diabetes mellitus and Parkinson disease. Neurology. 2018;90(19):e1654-e1662.

29. Vidal-Martinez G, Yang B, Vargas-Medrano J, Perez RG. Could a-Synuclein Modulation of Insulin and Dopamine Identify a Novel Link Between Parkinson's Disease and Diabetes as Well as Potential Therapies?. Front Mol Neurosci. 2018;11:465.

30. Wild S, Roglic G, Green A, Sicree R, King H. Global prevalence of diabetes: estimates for the year 2000 and projections for 2030. Diabetes Care. 2004;27(5):1047-1053.

31. Gioia G, Salducci M. Medical and legal aspects of telemedicine in ophthalmology. Rom J Ophthalmol. 2019;63(3):197-207.

32. Liu Y, Torres DA, Benkert R. Scaling Up Teleophthalmology for Diabetic Eye Screening: Opportunities for Widespread Implementation in the USA. Curr Diab Rep. 2019;19:74.

33. Salongcay RP, Silva PS. The Role of Teleophthalmology in the Management of Diabetic Retinopathy. Asia Pac J Ophthalmol. (Phila). 2018;7(1):17-21.

34. Liesenfeld B, Kohner E, Piehlmeier W, et al. A telemedical approach to the screening of diabetic retinopathy: digital fundus photography. Diabetes Care. 2000;23:345-348.

35. Yu J-G, Feng Y-F, Xiang Y, Huang J-H, Savini G, Parisi V, et al. Retinal nerve fiber layer thickness changes in Parkinson disease: a meta-analysis. PLoS One. 2014;9(1):e85718. 